\title{
Evolution of the dust mass loss with luminosity along the giant branch of the globular cluster 47 Tucanae $^{\star}$
}

\author{
A. Ramdani ${ }^{1,2}$ and A. Jorissen ${ }^{2, \star \star}$ \\ 1 Département de Physique, Université d'Oujda, Maroc \\ 2 Institut d'Astronomie et d'Astrophysique, Université Libre de Bruxelles, CP 226, Boulevard du Triomphe, \\ 1050 Bruxelles, Belgium
}

Received 4 December 2000 / Accepted 7 March 2001

\begin{abstract}
The present paper investigates the properties of the dust mass loss in stars populating the giant branch of the globular cluster 47 Tuc, by combining ISOCAM and DENIS data. Raster maps of 5 fields covering areas ranging from $4 \times 4$ to $15 \times 15 \operatorname{arcmin}^{2}$ at different distances from the center of the cluster have been obtained with ISOCAM at $11.5 \mu \mathrm{m}$ (LW10 filter). The covered fields include most of the red variables known in this cluster. A detection threshold of about $0.2 \mathrm{mJy}$ is achieved, allowing us to detect giant stars at $11.5 \mu \mathrm{m}$ all the way down to the horizontal branch. No dust-enshrouded asymptotic giant branch stars have been found in the observed fields, contrary to the situation encountered in LMC/SMC globular clusters with larger turnoff masses.

The color index [12] - [2] (based on the ISO $11.5 \mu \mathrm{m}$ flux and on the DENIS $K_{\mathrm{s}}$ magnitude) is used as a diagnostic of dust emission (and hence dust mass loss). Its evolution with luminosity along the giant branch reveals that dust mass loss is only present in $V 3$ (the only cluster Mira variable observed in the present study) and in $V 18$, a star presenting intermittent variability. This conclusion confirms the importance of stellar pulsations in the dust formation and ensuing mass loss.
\end{abstract}

Key words. globular clusters: 47 Tuc - stars: AGB and post-AGB - circumstellar matter - stars: mass loss infrared: stars

\section{Introduction}

Stellar mass loss is an essential ingredient in the modelling of several important astrophysical processes. It plays a key role in the chemical evolution of the Galaxy, by returning to the interstellar medium the ashes of the nuclear-burning processes that took place during the star's lifetime. In Asymptotic Giant Branch (AGB) stars, the mass loss rate is so large that the evolutionary timescale is controlled by mass loss rather than by nuclear burning. The general principles governing mass loss on the AGB are understood. Shock waves associated with the stellar pulsation push the gas far enough above the photosphere for dust to start condensing. Radiation pressure on the dust, when it couples to the gas, then provides the necessary thrust for matter to escape (e.g., Sedlmayr \& Winters 1997). A parametrization of the mass loss rate on the AGB, in terms of the fundamental stellar parameters $M, L, R$ and $Z$, is not available from empirical data (e.g., Zijlstra 1995), but Arndt et al. (1997) have proposed one based on computed

\footnotetext{
Send offprint requests to: A. Jorissen, e-mail: ajorisse@astro.ulb.ac.be

* Table 3 is only available in electronic form at the CDS via anonymous ftp to cdsarc.u-strasbg.fr (130.79.128.5) or via

http://cdsweb.u-strasbg.fr/cgi-bin/qcat?J/A+A/372/85

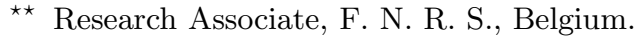

models (see below). The oldest among empirical mass-lossrate parametrizations is the Reimers formula (Reimers 1975; see Zijlstra 1995 and Sect. 11 of Habing 1996 for a discussion of the more recent parametrizations):

$\dot{M}\left(M_{\odot} \mathrm{y}^{-1}\right)=-4 \times 10^{-13} \eta \frac{\left(L / L_{\odot}\right)\left(R / R_{\odot}\right)}{\left(M / M_{\odot}\right)}$,

where $\eta$ is a numerical factor (introduced later by FusiPecci \& Renzini 1976; Mengel 1976) of the order of unity. Although the Reimers formula provides a fair representation of the mass loss rates measured for G, $\mathrm{K}$ and (nonlong-period-variable) $\mathrm{M}$ giants, it is well known that it predicts rates that are too low at the AGB-tip (e.g., de Jong 1983; Willson 1987; Bowen \& Willson 1991; Blöcker 1995). The final AGB masses obtained with a mass loss rate following the Reimers prescription all the way to the AGB-tip are too large to satisfy the initial-final mass relationship of Weidemann (1987). Therefore, Renzini (1981) suggested the existence of a short episode of strong mass loss close to the AGB-tip (dubbed "superwind"), with mass loss rates of the order of $10^{-5}$ to $10^{-4} M_{\odot} \mathrm{y}^{-1}$, whereas the Reimers formula predicts maximum mass loss rates of the order of $10^{-6} M_{\odot} \mathrm{y}^{-1}$. Luminous AGB stars with mass loss rates that large have since been found (e.g., de Jong 1983; Whitelock et al. 1991, 1994; Vassiliadis \& Wood 1993 and references therein), as well as empirical evidence for a sudden increase of the mass loss rate at 
the end of the AGB (e.g., Delfosse et al. 1997 and references therein). There has been much speculation concerning the nature and cause of the superwind. Willson (1981; her Figs. 5 and 6) and Bowen \& Willson (1991) argued that pulsation-driven mass loss in Mira variables accounts for the required superwind. On the other hand, Netzer \& Elitzur (1993) argued that the mass loss rate abruptly increases when the dust couples to the gas, thus causing the superwind phase. Detailed calculations of the AGB evolution, including all the ingredients (stellar structure, pulsation and shock waves, dust formation and coupling to the gas, radiative transfer) necessary to trigger mass loss in a self-consistent way are now being performed for carbon-rich Miras (Fleischer et al. 1992; Höfner et al. 1996; Sedlmayr \& Winters 1997; Winters et al. 2000) and reproduce the expected features of the superwind phase at the AGB-tip (Schröder et al. 1999). A parametrization of the mass loss rates, in terms of $T_{\text {eff }}, L$ and $M$, and based on the computed models, has been proposed by Arndt et al. (1997).

The sensitivity of mass loss to metallicity is one of the remaining question in the field. The low-metallicity behavior of dust- and pulsation-driven AGB mass loss has been investigated by Bowen \& Willson (1991) with the conclusion that low-metallicity AGB stars reach the same mass loss rates than their higher metallicity counterparts, albeit at higher luminosities. This prediction is basically confirmed by observations of mass-losing AGB stars in the Magellanic Clouds, which do not call for low mass loss rates at low $Z$ (see Zijlstra 1999; Olofsson 1999 for recent reviews).

Giant stars in globular clusters are ideal targets to address these questions. In particular, the variation of mass loss with luminosity may be easily followed, the impact on mass loss of the pulsation properties of the long-period variables may be investigated, as well as the impact of the subsolar metallicity characterizing globular-cluster stars.

Among globular clusters, 47 Tuc (NGC 104) offers several advantages. It hosts many well-studied long-period variables (Sawyer-Hogg 1973; Glass \& Feast 1973; Lloyd Evans 1974; Fox 1982; Frogel 1983), among which 4 Mira variables ( $V 1$ to $V 4)$ with periods ranging from 165 to $212 \mathrm{~d}$, and visual amplitudes ranging from 1.5 to 4 mag. It is nearby (distance modulus $=13.7$; Gratton et al. 1997) and has been the subject of many previous studies providing auxiliary data like bolometric magnitudes from $U B V I J H K L$ fluxes or assignments of its giant stars to either the AGB or the RGB (Frogel et al. 1981). The basic parameters of 47 Tuc are as follows: $[\mathrm{Fe} / \mathrm{H}]=-0.67$, age $\sim 10.5$ Gy (Gratton et al. 1997), turnoff mass $\sim 0.9 M_{\odot}$ (Hesser et al. 1987). Gillett et al. (1988) have performed a pointed observation of 47 Tuc with IRAS, and detected several sources at 12, 25 and $60 \mu \mathrm{m}$ (see Table 2). The use of ISOCAM onboard ISO (see Sect. 2) provides both a much better sensitivity $(0.2 \mathrm{mJy}$ instead of $10 \mathrm{mJy}$ at $12 \mu \mathrm{m}$ ) and a better angular resolution (a few arcseconds instead of 45 arcsec) than IRAS, and thus makes it possible to detect many more faint point sources than it was possible with IRAS.

The present study aims at monitoring the dust mass loss as a function of luminosity along the RGB and AGB in 47 Tuc. Relatively warm dust is easily detected from the flux excess that it produces at $12 \mu \mathrm{m}$. Several authors (e.g., Whitelock et al. 1994; Le Bertre \& Winters 1998; Jorissen \& Knapp 1998) have shown that there is a tight correlation (at least for oxygen-rich stars in the solar neighbourhood) between the mass loss rate and the $K-[12]$ color index, which may thus be used to trace the dust mass loss.

Another objective of the present study is to detect possible dust-enshrouded stars with no optical counterparts, as found by Tanabé et al. $(1997,1999)$ in globular clusters from the Magellanic Clouds.

\section{Observations}

Mosaic images of 5 fields at different distances from the center of the 47 Tuc cluster have been obtained with the ISOCAM camera on board ISO (see Kessler et al. 1996; Cesarsky et al. 1996, for descriptions of ISO and ISOCAM respectively) using the LW10 filter (closely matching the IRAS $12 \mu \mathrm{m}$ filter). The field positions have been chosen so as to survey most of the red variable stars in 47 Tuc. The Astronomical Observation Template (AOT) parameters for each of the fields are given in Table 1 . They were adjusted so as to detect sources as faint as possible without saturating on the brightest sources. These contradictory requirements made us select a number $N_{\text {obs }}$ of elementary integrations as large as possible (namely $N_{\text {obs }}=10$, to keep within the total allocated time) but with short integration times $\left(T_{\mathrm{int}}=2 \mathrm{~s}\right)$ and low gain $(\times 1)$. With this setup, the detector saturates for fluxes of the order of $0.8 \mathrm{Jy} /$ pixel, and remains linear up to about $0.3 \mathrm{Jy} /$ pixel. The pixel field of view was taken equal to either $3^{\prime \prime}$ (with the large field mirror) for the fields closest to the core, or $6^{\prime \prime}$ for fields farther out. The step size between two successive pointings of the mosaic was half the chip size (in either direction), i.e., $90^{\prime \prime}$ for the $6^{\prime \prime}$ pixel field of view or $45^{\prime \prime}$ for the $3^{\prime \prime}$ pixel field of view, to ensure 4 independent detections of the sources in the central region of the mosaic. The total integration time per source is therefore $4 \times N_{\text {obs }} \times T_{\text {int }}$. The very center of the cluster has not been observed to avoid saturation and crowding problems.

\section{Reductions}

\subsection{ISOCAM image}

The reduction of our ISOCAM data poses a special challenge as we are aiming at extracting sources as faint as possible in a field containing very bright (AGB) sources. Because of the strong detector memory effects, these sources will leave ghost images in the next pointings that ought not to be confused with faint sources. The PRETI routines allowed us to overcome at least some of these 
Table 1. Astronomical Observation Template parameters for the 47 Tuc observations (ISO program "AJORISSE 47TUC").

Filter

Date of observation
LW-10 (center $11.5 \mu \mathrm{m}$, range 8.0-15.0 $\mu \mathrm{m}$ ) 25-May-1996

\begin{tabular}{|c|c|c|c|c|c|}
\hline Field name & FIELD2 & $V 5$ & FIELD3 & FIELD4 & FIELD1 \\
\hline Planned targets & $V 3, V 7, V 11, V 15, V 18$ & $V 5$ & Lee \#1421 & $V 13$ & Lee \# 5529 \\
\hline Field center (J2000): $\alpha$ & $0 \mathrm{~h} 25 \mathrm{~m} 35 \mathrm{~s}$ & $0 \mathrm{~h} 25 \mathrm{~m} \mathrm{08s}$ & Oh $22 \mathrm{~m} 46 \mathrm{~s}$ & $0 \mathrm{~h} 22 \mathrm{~m} 47 \mathrm{~s}$ & $0 \mathrm{~h} 27 \mathrm{~m} \mathrm{0s}$ \\
\hline$\delta$ & $-72^{\circ} 04^{\prime} 30^{\prime \prime}$ & $-72^{\circ} 10^{\prime} 15^{\prime \prime}$ & $-72^{\circ} 18^{\prime} 00^{\prime \prime}$ & $-72^{\circ} 09^{\prime} 07^{\prime \prime}$ & $-71^{\circ} 55^{\prime} 00^{\prime \prime}$ \\
\hline Distance from cluster center (arcmin) & 6.9 & 7.2 & 14.5 & 7.4 & 16.7 \\
\hline Detector gain & 1 & 1 & 1 & 1 & 2 \\
\hline Pixel Field of View (arcsec) & 3 & 3 & 6 & 3 & 6 \\
\hline Number of pointings & $9 \times 9$ & $4 \times 4$ & $9 \times 9$ & $7 \times 7$ & $8 \times 8$ \\
\hline Total field of view $\left(\operatorname{arcmin}^{2}\right)$ & $7.6 \times 7.6$ & $3.85 \times 3.85$ & $15.2 \times 15.2$ & $6.1 \times 6.1$ & $13.7 \times 13.7$ \\
\hline Step between two pointings (arcsec) & 45 & 45 & 90 & 45 & 90 \\
\hline
\end{tabular}

problems. They were designed by Starck et al. (1999; see also Aussel et al. 1999) specifically for the detection of faint sources on ISOCAM images, after performing a careful removal of the cosmic ray glitches (both their shortand long-term effects) and correcting for the transient behaviour so as to provide a flat baseline.

The main steps of the reduction process are as follows. The default dark frame is first subtracted from the raw data (CIER files) using the CAM Interactive Analysis (CIA) package (Ott et al. 1997). The bad Col. 23 is replaced by the average of Cols. 22 and 24. The shortduration cosmic rays are identified from a $3 \sigma$ test on the short-term fluctuations of successive readouts and the affected readouts are masked. The long-term effects of cosmic rays are identified from their typical pattern in a multi-resolution median filter, and masked as well. Remaining long-term drifts (mainly due to the detector memory effect after passing on bright sources, and to the change of the ISOCAM configuration at the beginning of the observation) are suppressed by subtracting the baseline of each pixel's time history, obtained from the sequence of all the individual images.

The flat field is constructed from the median of the data themselves, since in the best cases, about $800(\sim 10 \times$ $9 \times 9)$ readouts are available per pixel. The detector transient is corrected by the "inverse method" developed by Abergel et al. (1996). In the present analysis, the fluctuations arising on top of the bright sources (probably caused by the jitter of the satellite) are often confused with short-term cosmic rays and unduly masked, resulting in a loss of useful readings for bright sources. This problem could possibly lead to underestimating the flux of bright sources, but the effect - if present - does not appear to be very severe, as will be seen from a comparison of our ISOCAM fluxes with previous IRAS measurements of the same sources (see Table 2).
The individual mosaic frames are then coadded into a single image. The source detection is performed on that image (Fig. 1) using a wavelet transform, which detects at each scale all pixels of the image above a given threshold $\sigma$ of the noise map (Starck et al. 1999). A good compromise between maximum sensitivity and minimum number of false detections (as compared to optical sources; see Sect. 3.3) is obtained with a detection threshold set at $5 \sigma$. More details about the criteria of inclusion of sources in the final source list are provided in Sect. 4.

The source fluxes are obtained by aperture photometry on the reduced image. The aperture used includes 12 pixels, and is the sum of the $3 \times 3$-pixels square centered on the source, plus 4 pixels exterior to this square and located in the middle of each side of the square.

The measured values were then multiplied by the factors $100 / 72$ (for the fields observed with a $3^{\prime \prime}$ pixel field-of-view; see Table 1) or 100/84.5 (for the fields observed with the $6^{\prime \prime}$ pixel field-of-view) to account for the point-spread-function (PSF) wing not falling on the selected aperture. Those factors were computed from the observed PSF provided by Okumura (ISOCAM PSF Report, 04/Nov/1998, available on the ISOCAM page http://www.iso.vilspa.esa.es/users/expl_lib/CAM -list.html).

The measured ADUs are converted to Jansky units using the conversion factor provided by the CIA software package (4.129 ADU/s/mJy/pix). The uncertainty on the $12 \mu \mathrm{m}$ flux is the quadratic average of the Poisson noise on the signal, and of the uncertainty associated with the PSF correction (3\%).

Because the reduction process is an intricated one implying many different steps, it is interesting to compare the ISOCAM fluxes with previous results obtained by Gillett et al. (1988) from pointed IRAS observations of 47 Tuc (Table 2). 
Table 2. Comparison of IRAS and ISOCAM $12 \mu \mathrm{m}$ fluxes. IRAS fluxes are from Gillett et al. (1988), and $K$ magnitudes from Frogel et al. (1981), Glass \& Feast (1973) or DENIS (Cioni et al. 2000). The variable name refers to Sawyer-Hogg (1973), and the four-digits number for non-variable stars refers to Lee (1977). The period $P$ and visual amplitude $\Delta V$ for the variable stars are taken from Sawyer-Hogg (1973) or Fox (1982).

\begin{tabular}{|c|c|c|c|c|c|c|c|c|c|c|}
\hline \multirow[t]{2}{*}{ Name } & \multirow{2}{*}{$\begin{array}{c}\text { Alternate } \\
\text { name }\end{array}$} & \multicolumn{2}{|c|}{$F(12)(\mathrm{Jy})$} & \multicolumn{3}{|c|}{$K$} & \multirow[t]{2}{*}{$F 12 / F 2$} & \multirow{2}{*}{$\begin{array}{l}P \\
(\mathrm{~d})\end{array}$} & \multirow{2}{*}{$\begin{array}{c}\Delta V \\
(\mathrm{mag})\end{array}$} & \multirow[t]{2}{*}{ Rem. } \\
\hline & & IRAS & ISOCAM & Frogel/Glass & DENIS & DENIS name & & & & \\
\hline$V 3$ & & 0.215 & 0.203 & $6.00-6.49$ & - & & $0.08-0.12$ & 192 & 4.15 & \\
\hline$V 5$ & & 0.028 & 0.038 & 7.47 & 7.35 & J002501.97-720929.9 & 0.056 & $45,60-70$ & 0.7 & \\
\hline$V 7$ & & - & 0.055 & 6.97 & 6.79 & J002528.42-720652.2 & 0.051 & $50-58$ & 0.7 & \\
\hline$V 13$ & & 0.034 & 0.049: & 7.65 & & & - & 40 & 0.7 & * \\
\hline$V 15$ & W300 & 0.041 & 0.043 & 7.27 & 7.03 & J002551.35-720703.8 & 0.052 & 38 & 0.2 & \\
\hline$V 11$ & W12 & - & 0.095 & 6.69 & 6.48 & J002506.64-720220.7 & 0.068 & 52:, 100 & 0.8 & \\
\hline$V 18$ & L168 & - & 0.105 & 7.45 & - & & 0.150 & & 0.3 & \\
\hline$V 11+V 18$ & & 0.235 & 0.200 & - & - & - & & & & \\
\hline 7701 & $R 17$ & - & 0.028 & 7.66 & 7.43 & J002532.43-721052.2 & 0.049 & & & \\
\hline 8745 & $R 18=V 16$ & - & 0.036 & 7.44 & 7.08 & J002529.74-721118.4 & 0.051 & & & \\
\hline $7701+8745$ & & 0.065 & 0.064 & - & - & & & & & \\
\hline$R 19$ & & 0.034 & 0.032: & & & & & & & $*$ \\
\hline$V 28$ & $L R 5$ & 0.078 & 0.071 & & & & & 40: & 0.4 & FIELD3, * \\
\hline & & & 0.065 & & & & & & & FIELD4 \\
\hline 2620 & & 0.018 & 0.021 & & & & & & & \\
\hline 5529 & & 0.020 & 0.022 & 7.92 & 7.78 & J002604.55-715324.6 & 0.048 & & & \\
\hline 6304 & & 0.031 & 0.032 & & 7.22 & J002738.23-715257.6 & 0.038 & & & \\
\hline 1421 & & 0.071 & 0.062 & 6.84 & 6.68 & J002330.55-722236.5 & 0.050 & & & \\
\hline 1601 & & 0.023 & 0.024 & & 7.69 & J002401.03-721513.0 & 0.044 & & & \\
\hline 1603 & & 0.026 & 0.018 & 7.94 & 7.85 & J002339.15-721639.9 & 0.042 & & & \\
\hline 1533 & & 0.026 & 0.013 & & 8.21 & J002357.90-721857.8 & 0.038 & & & \\
\hline
\end{tabular}

Remarks:

$V 13:$ The ISOCAM image is a blend of 3 sources, as seen from the Digital Sky Survey image. R19: The ISOCAM image is a blend of 2 sources, as seen from the Digital Sky Survey image. 1421: Lee (1977) suspects this star to be a LPV.

$V 28=L R 5=$ Lee \#2758.

The $12 \mu \mathrm{m}$ IRAS and ISOCAM fluxes generally agree within $25 \%$, which is satisfactory considering the fact that the filters are not identical and that some of the LPV sources may be variable at $12 \mu \mathrm{m}$ as well. The largest deviations are obtained for the composite source $V 11+V 18$ (18\%), and for $V 13(31 \%)$. The $12 \mu \mathrm{m}$ flux of $V 13$ is not reliable, as the ISOCAM image of $V 13$ is in fact a blend of 3 different stars that are seen separately on the Digital Sky Survey image (Sect. 3.3). Another discrepancy concerns $V 7$, which does not seem to have been detected by Gillett et al. (1988). This non-detection is surprising, since its flux at the time of our ISOCAM observation is similar to that of $V 15$ which was detected by Gillett et al. (1988). A possible explanation would be that $V 7$ was much fainter in the $12 \mu \mathrm{m}$ band at the time of the IRAS observation. This seems unlikely, however, since the ISOCAM flux for $V 7$ agrees with the prediction for a dust-free photosphere (see Fig. 3), so that a smaller IRAS flux would mean that $V 7$ was then underluminous at $12 \mu \mathrm{m}$.

Note that the star $V 28$ (=LR $5=$ Lee \#2758) is present on both FIELD3 and FIELD4. The ISOCAM fluxes from these two independent measurements differ by only $9 \%$ (Table 2 ), which provides an estimate of the internal consistency of the derived fluxes. 


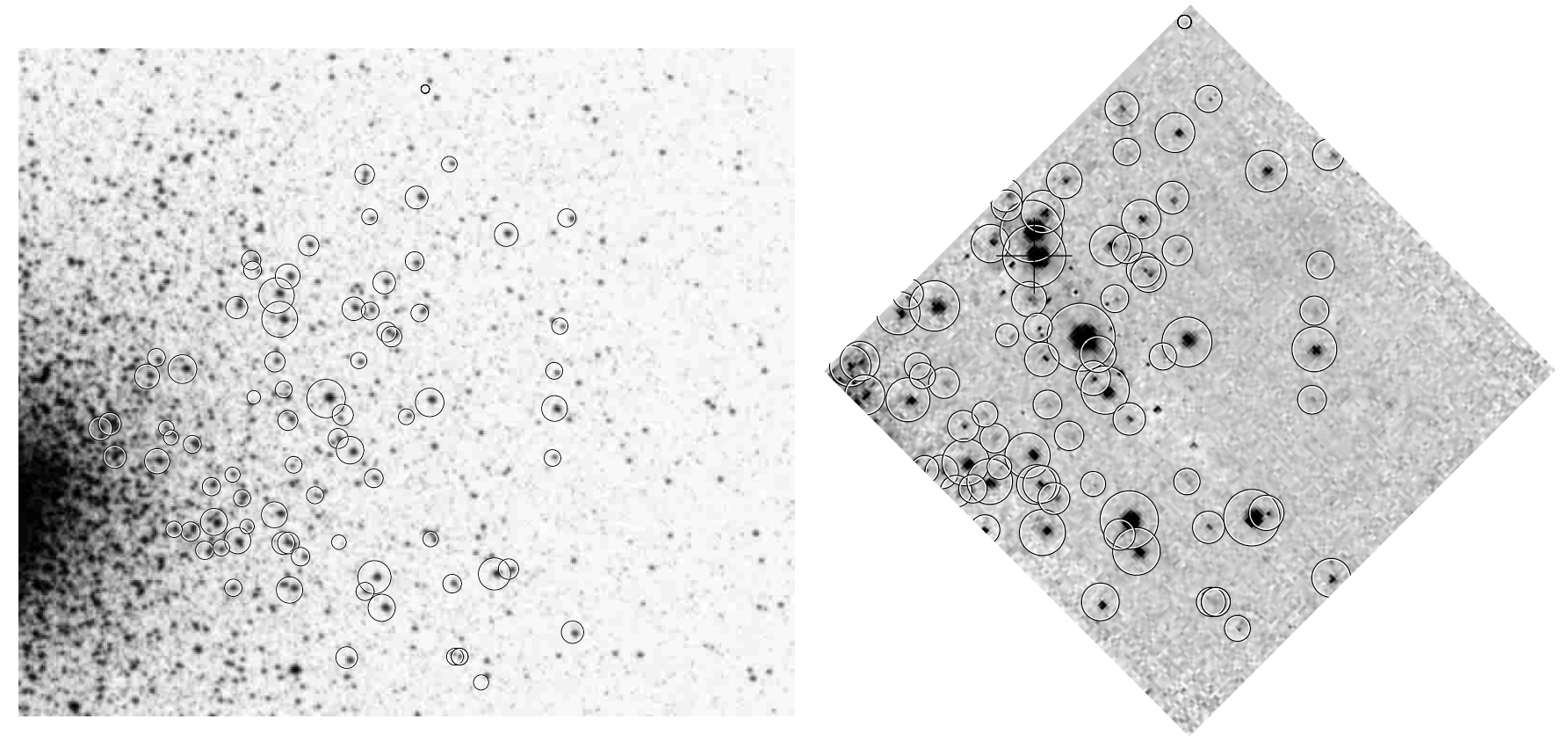

Fig. 1. Comparison of the Digital Sky Survey (left panel) and ISOCAM $12 \mu \mathrm{m}$ (right panel) images corresponding to FIELD2. The $12 \mu \mathrm{m}$ sources are identified by circles with a radius proportional to their $12 \mu \mathrm{m}$ flux (the variable $V 18$ discussed in the text is identified by the cross on the right panel). East is to the right, North is up. The field size of the Digital Sky Survey image is $12 \times 12 \operatorname{arcmin}^{2}$.

\subsection{DENIS data}

As the $K_{\mathrm{s}}$ magnitude provided by the Deep Near Infrared Survey (DENIS; see Epchtein 1998) saturates at $K_{\mathrm{s}}=6.5$ (Epchtein 1998), $K$ magnitudes for the brightest sources detected on the ISOCAM images are taken from Frogel et al. (1981) or from Glass \& Feast (1973). For the fainter sources, data from DENIS have been used, as published by Cioni et al. (2000) for fields around the Small Magellanic Cloud.

The cross-identification between ISOCAM and DENIS sources has been performed by overplotting their positions on the ISOCAM image. In most cases, an almost constant offset separated the positions of a given source in the two datasets. In some cases, however, an atypical offset whose origin is currently unknown had to be applied, especially for the brightest sources ( $V 7$ and $V 15$ ). The cross-identification of these sources leaves little doubt, as they generally correspond to the brightest sources in the area. Examples of cross identifications are listed in Table 2.

The variable $V 3$ is the only one to vary by a large factor (about $4 \mathrm{mag}$ in $V$ and $0.5 \mathrm{mag}$ in $K$; SawyerHogg 1973; Frogel et al. 1981). The other variables have amplitudes smaller than $0.9 \mathrm{mag}$ in $V$ (Lloyd Evans 1974; Fox 1982). Therefore, they are not expected to vary by more than $0.1 \mathrm{mag}$ in $K$. The average deviation $K$ (Frogel) $-K_{\mathrm{s}}$ (DENIS) is $0.21 \mathrm{mag}$ (excluding $V 3$ ).

\subsection{Optical data}

Lee (1977) and Chun \& Freeman (1978) provide $U B V$ data for most of the fields surveyed by the present ISOCAM observations. The assignment of a given giant star to either the RGB or AGB is possible from the $(V, B-V)$ diagram presented in Fig. 2 for the stars detected at $12 \mu \mathrm{m}$.

Optical images from the Digital Sky Survey were used to identify the optical counterparts of the infrared sources, along with the optical positions provided by Tucholke (1992) and its cross-identifications with the catalogues of Lee (1977) and Chun \& Freeman (1978). The left panel of Fig. 1 identifies the $12 \mu \mathrm{m}$ sources on the optical image for FIELD2. Basically all sources brighter than $V=14$ (i.e. $M_{V}<0.3$ for the distance modulus $V-M_{V}=13.7$ of 47 Tuc) are detected at $12 \mu \mathrm{m}$, corresponding to a completeness threshold of about $0.7 \mathrm{mJy}$ including all the giants down to the horizontal branch (Fig. 2).

\section{Final ISOCAM source list and absence of dust-enshrouded AGB stars}

The final list of ISOCAM $12 \mu \mathrm{m}$ sources obtained from the present observations (Table 3) has been obtained in the following way. A first list of candidate sources is provided by the $5 \sigma$ detections as described in Sect. 3.1. Obviously spurious sources in that list, resulting from detector memory effects that were not totally eliminated by the reduction process, have been discarded. A few faint sources observed only on the ISOCAM images, but neither on the optical (Sect. 3.3) nor on the DENIS $K$ images (Sect. 3.2) have been discarded as well. Table 3 lists the infrared 


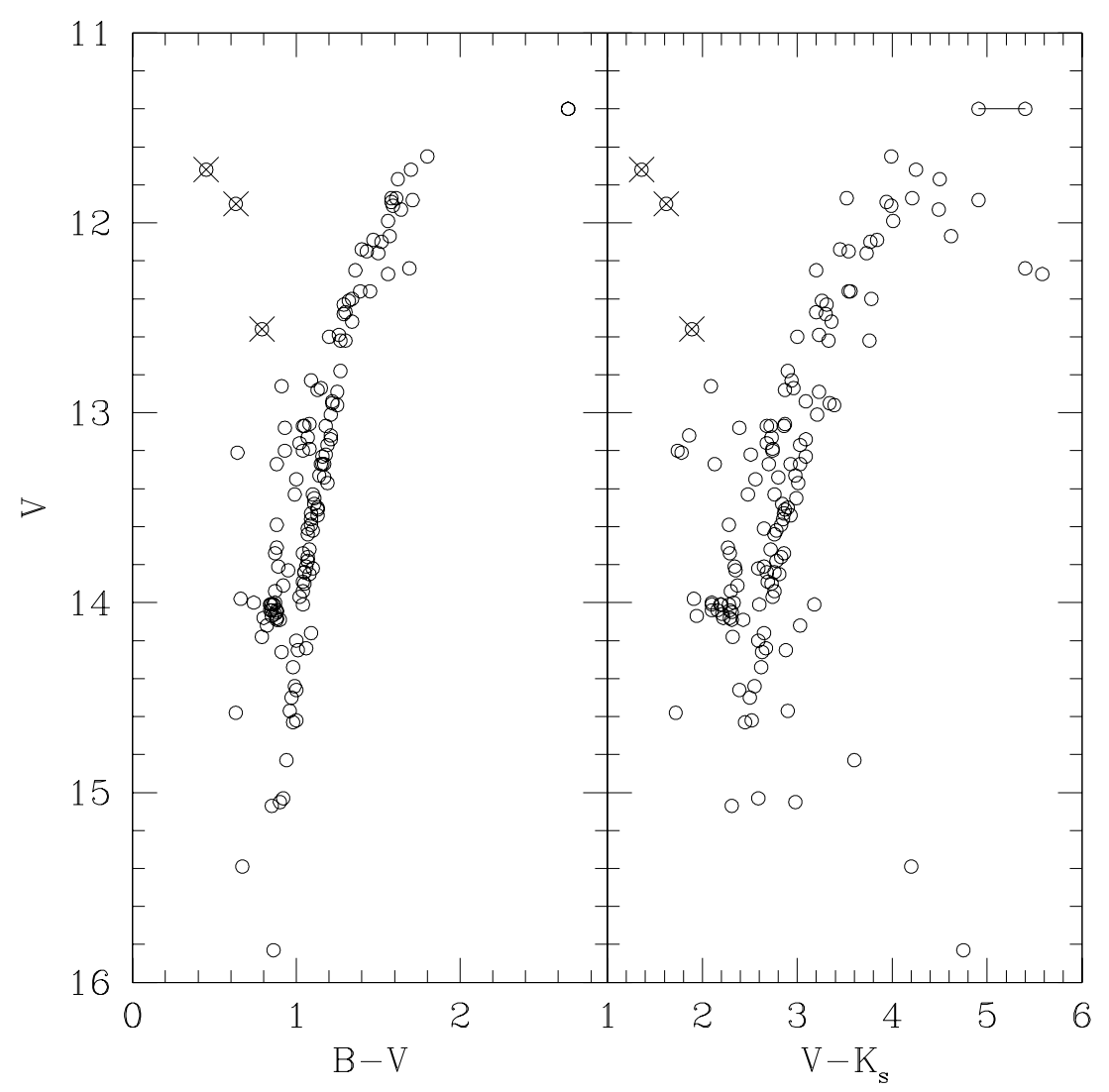

Fig. 2. The $(V, B-V)$ (left panel) and $\left(V, V-K_{\mathrm{s}}\right)$ (right panel) diagrams for the stars detected at $12 \mu \mathrm{m}$ and with $B, V$ magnitudes available from Lee (1977) or Chun \& Freeman (1978), and $K_{\mathrm{s}}$ from Table 3. The outliers (non-members?) 1321, 1412, 6209 are denoted by crosses.

Table 3. List of sources detected at $12 \mu \mathrm{m}$. The first column provides the source identifier from Lee (1977), Chun \& Freeman (1978) or Tucholke (1992) (the latter being referred to by "tuc" followed by a 4-digit number). The next three columns list the $V$ magnitude from Lee (1977) or Chun \& Freeman (1978), the DENIS $K_{\text {s }}$ magnitude (except for $V 3$, where $K$ is taken from Frogel et al. 1981), and the $V-K$ index. The fifth column lists the magnitude at $12 \mu \mathrm{m}$, derived from the flux in Jy $(F 12)$ using the relation $[12]=-2.5 \log F 12 / 28.3$. The $F 12 / F 2$ ratio is listed next, with $F 2(\mathrm{Jy})=665 \times 10^{-0.4 K_{\mathrm{s}}}$. The uncertainty $\sigma(F 12 / F 2)$ on $F 12 / F 2$ is given in Col. 7 , and includes only the uncertainty coming from $F 12$ (see Sect. 3.1). Column 8 indicates the field number to which the star belongs, and the last column contains remarks (like non-membership, based on an outlying position in the $(V, B-V)$ or $\left(K_{\mathrm{s}}, V-K_{\mathrm{s}}\right)$ diagrams). Table 3 is only available in electronic form at the CDS via anonymous ftp to cdsarc.u-strasbg.fr (130.79.128.5) or via http://cdsweb.u-strasbg.fr/cgi-bin/qcat?J/A+A/372/85

sources, along with their optical identifiers, ordered by decreasing optical brightness.

The comparison of the optical images with our list of ISOCAM sources makes it possible to look for infrared sources with no optical counterparts. As can be seen in Fig. 1, there are none in FIELD2. The only source with no optical counterpart found in the present survey is the active X-ray galaxy X3 (Verbunt \& Hasinger 1998) observed in FIELD3. Its $12 \mu \mathrm{m}$ flux amounts to $6.5 \mathrm{mJy}$.

One of the important results obtained by the present study is thus the absence of bright IR sources without optical counterparts, that could be associated with dustenshrouded $A G B$ stars. This result may be used to set an upper limit to the lifetime of dust-enshrouded AGB stars. To do so, the number of horizontal-branch stars in the
$529 \operatorname{arcmin}^{2}$ area covered by the fields listed in Table 1 is first evaluated. For that purpose, positions were taken from the catalogue of Tucholke (1992), with $V$ and $B$ magnitudes from Lee (1977) and Chun \& Freeman (1978). Stars on the horizontal branch were selected on the basis of their magnitude and color index satisfying the conditions $0.75 \leq B-V<0.94$ and $13.9 \leq V<14.2$. With 185 stars fulfilling these criteria, the fraction of obscured AGB stars to horizontal-branch stars turns out to be less than $1 / 185$. Adopting $1.23 \times 10^{8} \mathrm{y}$ for the lifetime of stars with $\left(M / M_{\odot}, Z\right)=(0.8,0.004)$ on the horizontal branch (Girardi et al. 2000), this fraction turns into an upper bound of $6.6 \times 10^{5} \mathrm{y}$ for the duration of the obscured AGB phase. Extrapolating to the whole cluster the absence of obscured AGB stars in the surveyed fields does not make 
this limit much more constraining, since the catalogue of Tucholke (1992) contains only about twice as many stars on the horizontal branch in the whole cluster. A limit of a few $10^{5} \mathrm{y}$ is not very meaningful, since the duration of the high mass-loss episode ("superwind") necessary to produce dust-enshrouded AGB stars appears to be of the order of a few $10^{4} \mathrm{y}$, according to models of mass-losing AGB stars computed by Schröder et al. (1999). Observations of the structure of the $\mathrm{CO}$ shell around the post-AGB star AFGL 618 (Meixner et al. 1998) and around some OH/IR objects (Delfosse et al. 1997) point towards a duration of a few $10^{3} \mathrm{y}$ or even less for the superwind phase [the OH/IR objects observed by Delfosse et al. 1997 are, however, probably rather massive ( $>5 M_{\odot}$ ?) and thus not representative of the AGB stars found in globular clusters].

The brevity of the corresponding evolutionary stage may not be, however, the only factor accounting for the absence of obscured AGB stars in 47 Tuc, since despite similarly adverse statistical expectations, Frogel et al. (1990) and Tanabé et al. $(1997,1999)$ nevertheless found such stars in three different globular clusters belonging to the SMC (NGC 419) or to the LMC (NGC 1783 and NGC 1978). These clusters belong to classes SWB IVVI (Searle et al. 1980) and are characterized by turnoff masses 1.5-1.6 $M_{\odot}$ (Nishida et al. 2000), much larger than the turn-off mass of 47 Tuc $\left(\sim 0.9 M_{\odot}\right)$, and ages in the range 1.6-2.0 Gyr. The obscured AGB stars found by Tanabé et al. $(1997,1999)$ have $M_{\text {bol }}=-4.9--5.0$, somewhat larger than the bolometric magnitudes of the variable stars at the AGB tip in 47 Tuc, which have $M_{\text {bol }}$ between -3.4 at minimum and -4.7 at maximum (Frogel et al. 1981). The larger initial masses of AGB stars in the clusters where dust-enshrouded stars have been found is probably the key to the existence of such stars in the LMC/SMC globular clusters and not in 47 Tuc. Habing (1996) has reviewed the evidence indicating that larger initial masses imply higher mass-loss rates on the AGB, which in turn imply thicker dust shells [see e.g., Eq. (2b) of Le Sidaner \& Le Bertre (1993) or Bedijn (1987)]. According to the spectral energy distributions computed by Bedijn (1987; his Fig. 4), dust-enshrouded stars have optical depths at $9.7 \mu \mathrm{m}$ in excess of $\sim 5$, and require mass loss rates in excess of about $10^{-5} M_{\odot} \mathrm{y}^{-1}$ (for typical values of the other relevant parameters), i.e., in the "superwind" regime. Schröder et al. (1999) have shown that AGB stars with masses lower than $1.1 M_{\odot}$ never reach large enough luminosities to drive a dustinduced superwind, and thus have no chance to become dust-enshrouded. Finally, the luminosity function of obscured AGB stars in the field of the LMC rises steeply at $M_{\mathrm{bol}} \sim-4.5$ (van Loon et al. 1999), which is about where the AGB ends in 47 Tuc (Frogel et al. 1981). Therefore, all available evidence points towards the low turnoff mass of 47 Tuc as being the main cause for its lack of dustenshrouded stars, due to the absence of a superwind phase in low-mass AGB stars.

\section{The F12/F2 index: Dust mass loss as a function of luminosity}

As indicated in Sect. 1, the ratio $F 12 / F 2$ of the 12 to $2 \mu \mathrm{m}$ fluxes is expected to be a good indicator of dust mass loss. Figure 3 presents the $F 12 / F 2$ vs. $K$ diagram for all the sources detected at 2 and $12 \mu \mathrm{m}$. As indicated in Sect. 3.1, the error bars reflect the Poissonian noise on the signal, and the uncertainty on the PSF-wing correction. To fix the ideas, the $F 12 / F 2$ ratio expected for dust-free stellar atmospheres has been estimated from the stellar atmosphere models of Plez et al. (1992) and Bessell et al. (1998). Models of solar metallicity have been used, as only those are available over the whole temperature range covered by the observed stars. Nevertheless, a comparison between $F 12 / F 2$ ratios obtained from $\left(T_{\text {eff }}=3800 \mathrm{~K}\right.$, $\log g=-0.5$ ) models of $[\mathrm{Fe} / \mathrm{H}]=0$ and -0.6 (yielding $F 12 / F 2=0.046$ and 0.045 , respectively) reveals that metallicity has a negligible impact on the $F 12 / F 2$ ratio (at least for those model stars with no mass loss).

The $F 12 / F 2$ ratio for most of the stars is thus consistent with the value predicted for dust-free photospheres (Fig. 3). Only the bright LPV star V3 (the only Mira in Fig. 3, with $P=192 \mathrm{~d}$ ) and, more surprisingly the low-amplitude variable $V 18$, do exhibit $12 \mu \mathrm{m}$ excesses. A marginal excess may be present as well for the irregular variable $V 11$. The exact value of the excess is, however, difficult to assess for $V 3$, as this Mira variable exhibits 0.5 -mag variations in the $K$ band (Frogel et al. 1981), and very likely varies as well in the $12 \mu \mathrm{m}$ band $^{1}$. The $F 12 / F 2$ ratio of $V 3$ should therefore ideally be derived from 2 and $12 \mu \mathrm{m}$ fluxes obtained at the same epoch, which is unfortunately not the case for the value displayed in Fig. 3. It is very likely, though, that $V 3$ does exhibit at least some excess at $12 \mu \mathrm{m}$.

The $12 \mu \mathrm{m}$ excess observed for $V 18$ is more surprising (there is no doubt about its reality, as may be assessed from Fig. 1), especially when compared to the absence of a similar excess for the stars $V 7$ and $V 15$, which are located slightly above $V 18$ on the giant branch. The origin of the difference between the $F 12 / F 2$ ratios of variable stars of comparable luminosities must probably be searched for in their variability properties. Both $V 11$ and $V 18$ are noteworthy in that respect. Fox (1982) notes that the irregular variable $V 11$ possibly changed its period (and pulsation mode?) from about $100 \mathrm{~d}$ at the time of Lloyd Evans' observations (Lloyd Evans 1974) to 52 d about 10 y later. Similarly, Fox (1982) suggests that $V 18$ may be of the type of variable that switches on and off intermittently.

\footnotetext{
${ }^{1}$ Variations in the $12 \mu \mathrm{m}$ IRAS fluxes by factors as large as 1.6 have been reported by Jorissen \& Knapp (1998) for some S-type Mira variables. The M-type Mira Z Cyg also exhibits variations by a factor of 2 at $12 \mu \mathrm{m}$ as revealed by ISO SWS spectra (Onaka et al. 1999). Finally, lightcurves in the $N$ band of O-rich Miras (Le Bertre 1993) reveal variations by as much as 1 mag (i.e., a factor of 2.5). These variations may be accounted for by the underlying luminosity and temperature variations of the Mira variable.
} 


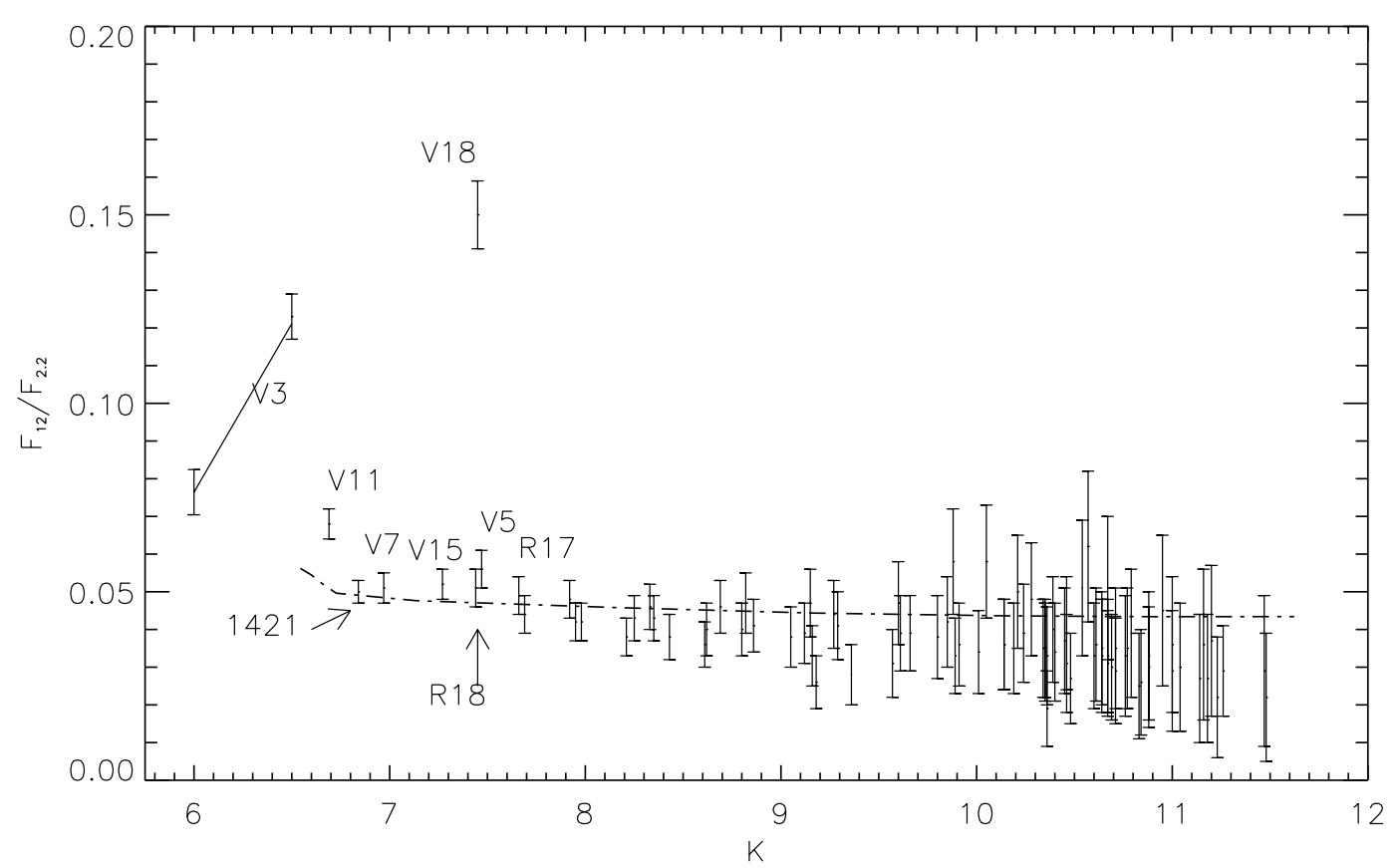

Fig. 3. The $F 12 / F 2$ vs. $K$ diagram for stars detected at 2 and $12 \mu \mathrm{m}$, with the variable stars identified. The conversion from $K$ magnitudes to fluxes was performed by adopting a zero-magnitude flux of 665 Jy, following Fouqué et al. (2000). Note that the distance modulus of 47 Tuc is 13.7 (Gratton et al. 1997). The oblique segment for $V 3$ translates its variability in the $K$ band only (see Table 2), as existing data do not allow yet to estimate its variability range at $12 \mu \mathrm{m}$. The dot-dashed line corresponds to the $F 12 / F 2$ ratio derived from dust-free model atmospheres (see text).

In the same vein, Tucholke (1992) lists two different $B$ magnitudes for $V 18$ ( $B=17.4$ and 13.5 for entries 2197 and 2198, respectively, which have exactly the same coordinates); they probably indicate that strong variations occurred over the time span covered by Tucholke's plates. It would be tempting to relate this behaviour with the sudden ejection of dust shells causing the star to brighten up at $12 \mu \mathrm{m}$. A similar phenomenon has been proposed for semi-regular variable stars by Ivezić \& Knapp (1999). Note that the large $F 12 / F 2$ ratio of $V 18$ cannot be ascribed to variations in the $K$ band, since the $K_{\mathrm{s}}$ magnitude estimated from the raw DENIS frame ${ }^{2}$ amounts to 7.33, as compared to 7.41 from the Two Micron All Sky Survey (Skrutskie 1997) and 7.44 obtained almost $20 \mathrm{y}$ earlier by Frogel et al. (1981). Despite the fact that $V 28$ is also suspected by Fox (1982) of being an intermittent variable, this variable star does not, however, show large excesses at $12 \mu \mathrm{m}(F 12 / F 2=0.067$ with $K=6.99$ from Fox 1982).

Nevertheless, Fig. 3 shows that the dust mass loss does not increase gradually along the AGB, but that it appears suddenly as the AGB star becomes strongly variable. The mass loss rates of the dusty AGB stars in 47 Tuc is not

\footnotetext{
2 Aperture photometry has been performed on the DENIS frame, and the zero-point has been estimated from a linear regression between the raw DENIS magnitudes and the $K$ magnitudes from Frogel et al. (1981) for the stars in common between the two samples. The slope of the regression line amounts to 1.013 and the maximum relative deviation is $2 \%$.
}

expected to be quite large, though. A blind application of the empirical relationship between the mass loss rate and the $K-[12]$ color $^{3}$ (Le Bertre \& Winters 1998) yields a total mass loss rate lower than $10^{-8} M_{\odot} \mathrm{y}^{-1}$ for $V 18$ ! In fact, this relationship applies to solar-metallicity stars and to the regime of dust-driven winds, when the dust is abundant enough to couple to the gas. Neither condition is satisfied for the LPVs in 47 Tuc. Netzer \& Elitzur (1993) have shown that the coupling between gas and dust is not achieved below mass loss rates of a few $10^{-8} M_{\odot} \mathrm{y}^{-1}$ (see also Fig. 20 of Jorissen \& Knapp 1998). Such a low value for the mass loss rate of $V 18$ is also confirmed by the application of a simple dust radiative-transfer model encapsulated in Eq. (6) of Frogel \& Elias (1988, as corrected by Eq. (17) of Jura \& Kleinman 1992). Adopting for $V 18$ $F_{12}$ (shell) $=0.07 \mathrm{Jy}$ (see Table 2 , after subtraction of the photospheric flux), $v=4 \mathrm{~km} \mathrm{~s}^{-1}$ for the dust outflow velocity (according to Fig. B1 of van Loon 2000 for variable stars with short periods), $T_{\text {eff }}=3680 \mathrm{~K}$ and $M_{\mathrm{bol}}=-2.96$ (Frogel et al. 1981) yielding $R=85 R_{\odot}$, and a grain cross section per unit mass $\kappa=2.310^{3} \mathrm{~cm}^{2} \mathrm{~g}^{-1}$ at $12 \mu \mathrm{m}$ for astronomical silicates with a density of $3.3 \mathrm{~g} \mathrm{~cm}^{-3}$ (Draine \& Lee 1984) yields $\dot{M}=\psi \times 2 \times 10^{-11} M_{\odot} \mathrm{y}^{-1}$, where $\psi$ is the gas-to-dust ratio. With $\psi$ of the order of $10^{3}$,

${ }^{3} K-[12]=-2.5 \log \left[\left(F_{2} / F_{12}\right)\left(F_{12}(0) / F_{2}(0)\right)\right]$. In the definition of the color index $K-[12], F_{2}(0)=665 \mathrm{Jy}$ and $F_{12}(0)=28.3 \mathrm{Jy}$ are normalization factors ensuring that stars with no dust excess have $K-[12]$ indices close to 0 (Fouqué et al. 2000; IRAS Explanatory Suppl. 1988). 
as expected for envelopes with metallicities of $1 / 5$ the solar value (solar-metallicity oxygen-rich envelopes typically have $\psi \sim 200$; e.g., van der Veen 1989; Justtanont et al. 1994), a mass loss rate of the order of $10^{-8} M_{\odot} \mathrm{y}^{-1}$ is indeed obtained. Incidentally, the $K-[12]$ index of 1.37 for $V 18$ appears quite small in comparison with mass-losing AGB stars in the solar neighbourhood, where $K-[12]$ falls in the range $2-6$ (corresponding to $0.27 \leq F 12 / F 2 \leq 11$ ). This result is consistent with the conclusion of Sect. 4 that the low-mass AGB stars of 47 Tuc never reached luminosities high enough to trigger dust-driven winds.

In any case, the fact that $12 \mu \mathrm{m}$-excesses are observed only for the LPV $V 3$, for the intermittent variable $V 18$ and possibly for the period-changing variable $V 11$, is an indication that stellar pulsations play a key role in triggering dust mass loss. Frogel \& Elias (1988) reached the same conclusion, based on the presence of $10 \mu \mathrm{m}$ excesses only in globular-cluster LPV stars and not in non-LPV cluster giants. The mass loss-pulsation connection is actually already well-documented, both on observational and theoretical grounds. A correlation between the mass loss rate and the pulsation period or amplitude of variation in the $K$ band has been noticed by various authors (Whitelock et al. 1987, 1994 for O-rich Miras; Claussen et al. 1987 and Groenewegen et al. 1998 for C-rich Miras; Vassiliadis \& Wood 1993 for a mixture of both). The various model calculations of mass loss in AGB stars have also shown the key importance of pulsations for triggering the mass loss process (see the discussion in Sect. 1).

\section{Conclusion}

The main results of the present study are (i) the absence of dust-enshrouded AGB stars in the surveyed fields, (ii) the existence of excess emission at $12 \mu \mathrm{m}$ due to circumstellar dust only for large-amplitude (or intermittent) variable stars.

The intermittent variable $V 18$ appears to have an excess at $12 \mu \mathrm{m}$ much larger than stars of similar luminosities on the giant branch. A long-term monitoring of this star spanning several years and covering the optical and infrared bands would be of great interest to identify the origin of its anomalous properties.

Acknowledgements. M. Groenewegen is thanked for his help in the early phase of this project. D. Elbaz and H. Aussel provided invaluable help with the reduction of the data. The ISOCAM data presented in this paper was analysed using $C I A$, a joint development by the ESA Astrophysics Division and the ISOCAM Consortium. The ISOCAM Consortium is led by the ISOCAM PI, C. Cesarsky.

\section{References}

Abergel, A., Bernard, J. P., Boulanger, F., et al. 1996, A\&A, 315, L329

Arndt, T. U., Fleischer, A. J., \& Sedlmayr, E. 1997, A\&A, 327, 614
Aussel, H., Cesarsky, C. J., Elbaz, D., \& Starck, J. L. 1999, A\&A, 342, 313

Bedijn, P. J. 1987, A\&A, 186, 136

Bessell, M. S., Castelli, F., \& Plez, B. 1998, A\&A, 333, 231

Blöcker, T. 1995, A\&A, 297, 727

Bowen, G. H., \& Willson, L. A. 1991, ApJ, 375, L53

Cesarsky, C., Abergel, A., Agnese, P., et al. 1996, A\&A, 315, L32

Chun, M. S., \& Freeman, K. C. 1978, AJ, 83, 376

Cioni, M.-R., Loup, C., Habing, H. J., et al. 2000, A\&AS, 144, 235

Claussen, M. J., Kleinmann, S. G., Joyce, R. R., \& Jura, M. 1987, ApJS, 65, 385

de Jong, T. 1983, ApJ, 274, 252

Delfosse, X., Kahane, C., \& Forveille, T. 1997, A\&A, 320, 249

Draine, B. T., \& Lee, H. M. 1984, ApJ, 285, 89

Epchtein, N. 1998, in The impact of near-infrared surveys on galactic and extragalactic astronomy, ed. N. Epchtein (Dordrecht, Kluwer Astrophysics and Space Science Library), 3

Fleischer, A. J., Gauger, A., \& Sedlmayr, E. 1992, A\&A, 266, 321

Fouqué, P., Chevallier, L., Cohen, M., et al. 2000, A\&AS, 141, 313

Fox, M. W. 1982, MNRAS, 199, 715

Frogel, J. A. 1983, ApJ, 272, 167

Frogel, J. A., Persson, S. E., \& Cohen, J. G. 1981, ApJ, 246, 842

Frogel, J. A., \& Elias, J. H. 1988, ApJ, 324, 823

Frogel, J. A., Mould, J., \& Blanco, V. M. 1990, ApJ, 352, 96

Fusi-Pecci, F., \& Renzini, A. 1976, A\&A, 46, 447

Gillett, F. C., de Jong, T., Neugebauer, G., Rice, W. L., \& Emerson, J. P. 1988, AJ, 96, 116

Girardi, L., Bressan, A., Bertelli, G., \& Chiosi, C. 2000, A\&AS, 141,371

Glass, I. S., \& Feast, M. W. 1973, MNRAS, 163, 245

Gratton, R. G., Fusi Pecci, F., Carretta, E., et al. 1997, ApJ, 491, 749

Groenewegen, M. A. T., Whitelock, P. A., Smith, C. H., \& Kerschbaum, F. 1998, MNRAS, 293, 18

Habing, H. J. 1996, A\&AR, 7, 97

Hesser, J. E., Harris, W. E., VandenBerg, D. A., et al. 1987, PASP, 99, 739

Höfner, S., Feuchtinger, M. U., \& Dorfi, E. A. 1996, A\&A, 297, 815

Ivezić, Ž., \& Knapp, G. R. 1999, in Asymptotic Giant Branch Stars, IAU Symp. 191, ed. T. Le Bertre, A. Lèbre, \& C. Waelkens, San Francisco, ASP, 395

IRAS Explanatory Supplement, 2nd edition 1988, Joint IRAS Science Working Group, Washington DC, US GPO

Jorissen, A., \& Knapp, G. R. 1998, A\&AS, 129, 363

Justtanont, K., Skinner, C. J., \& Tielens, A. G. G. M. 1994, ApJ, 435, 852

Jura, M., \& Kleinman, S. G. 1992, ApJS, 79, 105

Kessler, M. F., Steinz, J. A., Anderegg, M. E., et al. 1996, A\&A, 315, L27

Lee, S.-W. 1977, A\&AS, 27, 381

Le Bertre, T. 1993, A\&AS, 97, 729

Le Bertre, T., \& Winters, J. M. 1998, A\&A, 334, 173

Le Sidaner, P., \& Le Bertre, T. 1993, A\&A, 278, 167

Lloyd Evans, T. 1974, MNRAS, 167, 393

Meixner, M., Campbell, M. T., Welch, W. J., \& Likkel, L. 1998, ApJ, 509, 392 
Mengel, J. G. 1976, A\&A, 48, 83

Netzer, N., \& Elitzur, M. 1993, ApJ, 410, 701

Nishida, S., Tanabé, T., Nakada, Y., et al. 2000, MNRAS, 313, 136

Olofsson, H. 1999, in Asymptotic Giant Branch Stars, IAU Symp. 191, ed. T. Le Bertre, A. Lèbre, \& C. Waelkens, San Francisco, Astron. Soc. Pacific, 3

Onaka, T., de Jong, T., Yamamura, I., Cami, J., \& Tanabé, T. 1999, in The Universe as seen by ISO, ed. P. Cox, \& M. F. Kessler, ESA-SP 427, 381

Ott, S., Abergel, A., \& Altieri, B. 1997, in Astronomical Data Analysis Software and Systems VI. San Francisco, ed. G. Hunt, \& H. E. Payne, ASP Conf. Ser., 125, 34

Plez, B., Brett, J. M., \& Nordlund, A 1992, A\&A, 256, 551

Reimers, D. 1975, Mem. Soc. Roy. Sc. Liège, 6e Ser., 8, 369

Renzini, A. 1981, in Physical processes in red giants, ed. I. Jr. Iben, \& A. Renzini (Reidel, Dordrecht), 431

Sawyer-Hogg, H. 1973, Publ. DDO, 3, No. 6

Schröder, K.-P., Winters, J. M., \& Sedlmayr, E. 1999, A\&A, 349,898

Searle, L., Wilkinson, A., \& Bagnuolo, W. G. 1980, ApJ, 239, 803

Sedlmayr, E., \& Winters, J. M. 1997 in Stellar atmospheres: Theory and observations, ed. J. P. De Grève., R. Blomme, \& H. Hensberge (Berlin, Springer Verlag), 89

Skrutskie, M. F. 1997, Sky \& Telescope 94, 46

Starck, J. L., Aussel, H., Elbaz, D., Fadda, D., \& Cesarsky, C. 1999, A\&AS, 138, 365
Tanabé, T., Nishida, S., Matsumoto, S., et al. 1997, Nature, 385,509

Tanabé, T., Nishida, S., Nakada, Y., et al. 1999, in Asymptotic Giant Branch Stars, IAU Symp. 191, ed. T. Le Bertre, A. Lèbre, \& C. Waelkens, San Francisco, ASP, 573

Tucholke, H.-J. 1992, A\&AS, 93, 293

van der Veen, W. E. C. J. 1989, A\&A, 210, 127

van Loon, J. Th. 2000, A\&A, 354, 125

van Loon, J. Th., Groenewegen, M. A. T., de Koter, A., et al. 1999, A\&A, 351, 559

Vassiliadis, E., \& Wood, P. R. 1993, ApJ, 413, 641

Verbunt, F., \& Hasinger, G. 1998, A\&A, 336, 895

Weidemann, V. 1987, A\&A, 188, 74

Whitelock, P. A., Pottasch, S. R., \& Feast, M. W. 1987, in Late stages of stellar evolution, ed. S. Kwok, \& S. R. Pottasch (Kluwer, Dordrecht), 269

Whitelock, P., Feast, M., \& Catchpole, R. 1991, MNRAS, 248, 276

Whitelock, P., Menzies, J., Feast, M., et al. 1994, MNRAS, 267, 711

Winters, J. M., Le Bertre, T., Jeong, K. S., Helling, Ch., \& Sedlmayr, E. 2000, A\&A, 361, 641

Willson, L. A. 1987, in Late stages of stellar evolution, ed. S. Kwok, \& S. R. Pottasch (Kluwer, Dordrecht), 253

Zijlstra, A. A. 1995, ApSS, 224, 309

Zijlstra, A. A. 1999, in Asymptotic Giant Branch Stars, IAU Symp. 191, ed. T. Le Bertre, A. Lèbre, \& C. Waelkens, San Francisco, Astron. Soc. Pacific, 551 